\title{
Swelling of cellular solids: From conventional to re-entrant honeycombs
}

\author{
Ahmad Rafsanjani, ${ }^{1,2, a)}$ Dominique Derome, ${ }^{2, b)}$ Robert A. Guyer, ${ }^{3, \mathrm{c})}$ and Jan Carmeliet, ${ }^{2,4, d)}$ \\ ${ }^{1}$ Department of Civil, Environmental and Geomatic Engineering, ETH Zurich, CH-8093 Zurich, Switzerland \\ ${ }^{2}$ Laboratory for Building Science and Technology, Swiss Federal Laboratories for Materials Science and \\ Technology, EMPA, Ueberlandstrasse 129, CH-8600 Duebendorf, Switzerland \\ ${ }^{3}$ Department of Physics, University of Nevada, Reno, Nevada 89557, USA \\ ${ }^{4}$ Chair of Building Physics, ETH Zurich, Wolfgang-Pauli-strasse 15, CH-8093 Zurich, Switzerland
}

(Received 6 March 2013; accepted 13 May 2013; published online 29 May 2013)

\begin{abstract}
We find that, in two-dimensional periodic cellular solids, the hygro-expansion properties of the cell wall and the geometrical configurations of the lattice determine the effective swelling behavior of the medium. In this letter, we present the associated phase diagram for the swelling anisotropy of conventional and re-entrant honeycomb morphologies. The presented results are obtained numerically from a finite element based computational upscaling scheme. We show how the pattern of anisotropy in swelling behavior of cellular materials reverses when swelling is more important across or along the cell walls. (C) 2013 AIP Publishing LLC. [http://dx.doi.org/10.1063/1.4807844]
\end{abstract}

Cellular solids are broadly used for engineering applications such as cushioning, filtration, insulation, and lightweight sandwich cores and in many load-bearing systems. ${ }^{1}$ Understanding the mechanics of cellular materials in response to environmental stimuli is crucial for optimum material design. ${ }^{2}$ The swelling behavior of natural cellular materials is of practical interest since many biological materials experience environments with varying relative humidity. Sorption of water in the cell wall of cellular tissues results in moisture induced swelling strains and softening of the material. ${ }^{3,4}$ The hygro-expansion of wood, ${ }^{5}$ the ripening of fruits, ${ }^{6}$ and the complex movements in plants ${ }^{7,8}$ are all examples of the role of swelling in biological materials with cellular microstructure. Cellular solids are composed of a network of connected struts and a void space in between. The mechanics of honeycombs is initially studied by Gillis as an approximation to the basic unit of wood tracheids. ${ }^{9}$ Later Gibson et al. ${ }^{10}$ proposed a general model for estimating the effective mechanical properties of honeycombs where the cell walls deform due to bending. The effective elastic properties of re-entrant honeycombs are also studied by Kolpakov. ${ }^{11}$ The re-entrant mechanism occurs when an exterior tension applied in one direction leads to expansion in the other direction. ${ }^{12}$ The re-entrant honeycombs belong to a specific group of materials, called auxetics, which have an overall negative Poisson's ratio. The hygroelastic constitutive equation of a linearly elastic solid with moisture induced swelling can be expressed as

$$
\sigma_{i j}=C_{i j k l}\left(\varepsilon_{k l}-\beta_{k l} \Delta m\right),
$$

in which $\sigma_{i j}$ and $\varepsilon_{i j}$ are, respectively, second-order stress and strain tensors, $C_{i j k l}$ is the fourth-order stiffness tensor, $\beta_{i j}$ is

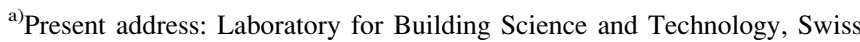
Federal Laboratories for Materials Science and Technology, EMPA, Ueberlandstrasse 129, CH-8600 Duebendorf, Switzerland. Electronic addresses: raahmad@ethz.ch and ahmad.rafsanjani@empa.ch

${ }^{b)}$ Electronic mail: dominique.derome@empa.ch

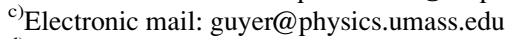

${ }^{\mathrm{d})}$ Electronic mail: carmeliet@arch.ethz.ch
}

the second-order tensor of hygro-expansion, and $\Delta m$ is the change in the moisture content. The expression for moisture induced swelling is formally similar to thermal expansion in which the moisture content plays the role of temperature. If the struts in a cellular material are composed of homogeneous isotropic material, the macroscopic thermal (or hygric) expansion is the same as that of the solid from which it is made. ${ }^{2}$ Lakes showed that arbitrary high thermal expansions can be achieved in cellular solids composed of bi-material ribs made of two bounded layers with differing thermal expansion coefficients. ${ }^{13,14}$ In a recent investigation, we showed that honeycombs composed of multi-layered cell walls with contrasting hygro-expansion coefficients exhibit anisotropic swelling behavior. ${ }^{15}$ In this letter, we present the anisotropy phase diagram for the swelling behavior of periodic honeycomb cellular materials from conventional to reentrant honeycombs in two dimensions. We describe the macroscopic behavior of periodic cellular solids with an energetically equivalent continuum following the micromechanics of solids. ${ }^{16}$ An efficient computational upscaling scheme is devised in the finite element package ABAQUS (Rising Sun Mills, Providence, RI, USA), and the effective swelling coefficients of honeycombs are computed. ${ }^{11}$

Figs. 1(a) and 1(b) show the honeycomb unit cells for $\theta>0^{\circ}$ and $\theta<0^{\circ}$, respectively. The geometrical parameters of the unit cells are cell wall lengths $h$ and $l$, cell wall thickness $t$, and shape angle $\theta$. The aspect ratio of the cell wall lengths is defined as $\xi=l / h$. The geometrical space of different honeycomb configurations as a function of $\theta$ and $\xi$ is shown in Fig. 1(c). It can be easily shown that when $\theta<0^{\circ}$, the possible domain should fall below the curve $\sin \theta$ $=-1 / 2 \xi$ in order to avoid the intersection of the struts. Our goal is to calculate the effective swelling behavior of the cellular materials from the elastic and the swelling properties of the cell walls. It is assumed that the swelling coefficients of the cell wall in parallel $(\|)$ and perpendicular $(\perp)$ directions are different. These two directions are marked in Fig. 1(a). The swelling normal to the cell wall (thickness expansion) is denoted with swelling coefficient $\beta_{\perp}$ while the swelling parallel to the cell wall (length expansion) is described with 

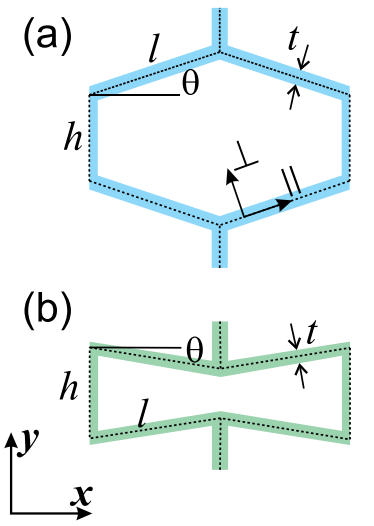

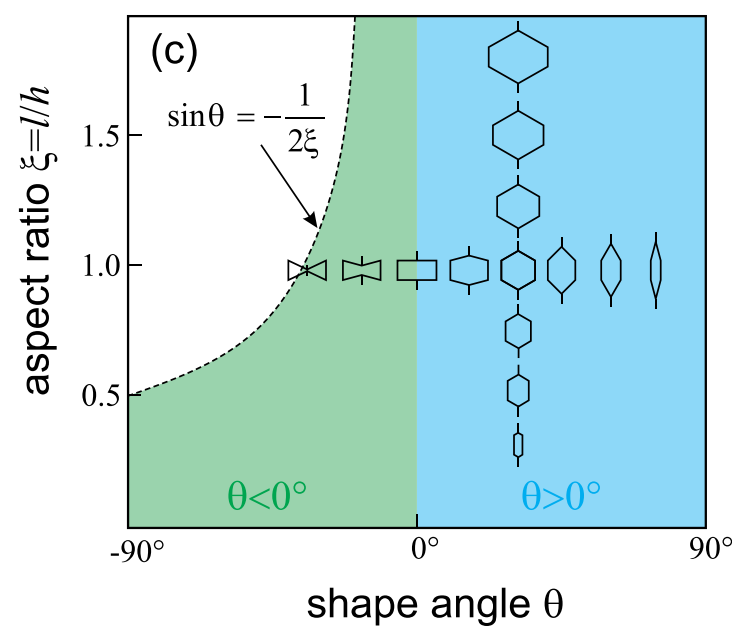

FIG. 1. Geometrical parameters of (a) conventional and (b) re-entrant honeycomb unit cells, (c) $\xi-\theta$ domain with examples of variation of the geometry of honeycombs with shape angle and aspect ratio. swelling coefficient $\beta_{\|}$. We focus on two specific cases in which the swelling of the cell wall is anisotropic, larger either in normal or parallel directions, for which we chose $\beta_{\|} / \beta_{\perp}=0.1$ and $\beta_{\|} / \beta_{\perp}=10$, respectively. This choice is motivated by the fact that in some natural cellular materials such as softwoods, the thickness expansion $\left(\beta_{\perp}\right)$ of the cell walls is much larger than the length expansion $\left(\beta_{\|}\right) .{ }^{17}$ Therefore, the macroscopic swelling anisotropy of the softwoods may be attributed in part to the anisotropy of the cell wall material. In order to achieve an anisotropy ratio of three as observed in earlywood cells of softwood, ${ }^{5}$ the swelling coefficient normal to the thickness $\beta_{\perp}$ should be roughly tenfold larger than swelling along the cell wall $\beta_{\|}{ }^{18}$ When cellular solids deform, the cell walls bend or undergo axial deformation. In both cases, the mechanical behavior is related to the elastic modulus of the cell walls in parallel direction, and the role of elastic modulus in normal direction is negligible. ${ }^{2}$ Therefore, we fixed the elastic properties of the cell wall to be isotropic with Young's modulus $E=1 \mathrm{GPa}$ and Poisson's ratio $\nu=0.3$. In all simulations, the thickness of the cell wall is $t / h=0.05$. The geometrical parameters are varied in the range $\theta=\left[-60^{\circ}, 75^{\circ}\right]$ and $\xi=[0.25,2.0]$. For each configuration, the associated directional swelling coefficient $\beta_{\varphi}$ is calculated according to relation

$$
\beta_{\varphi}=\beta_{x} \cos ^{2} \varphi+\beta_{y} \sin ^{2} \varphi+\beta_{x y} \sin \varphi \cos \varphi,
$$

where $\varphi$ is the directional angle with respect to $x$-axis (see Fig. 2) and $\beta_{x}=\beta_{11}, \beta_{y}=\beta_{22}$, and $\beta_{x y}=\beta_{12}$ are the components of in-plane swelling coefficient tensor which are obtained numerically from the upscaling scheme. In the whole range of geometrical parameters, from conventional to re-entrant honeycombs, we found $\beta_{x y} \simeq 0$. This happens because the geometry of the unit cells is symmetric with respect to $x$ and $y$ axes. The corresponding swelling anisotropy phase diagrams, grouping all the polar plots, for $\beta_{\|} / \beta_{\perp}=0.1$ and $\beta_{\|} / \beta_{\perp}=10$, are found in Figs. 2(a) and 2(b), respectively. If the behavior is isotropic, the resulting polar plot becomes a circle while the anisotropic swelling behavior generates a dumbbell shape. These polar plots show how the total macroscopic swelling strain varies at each directional angle $\varphi$. Based on these phase diagrams, the
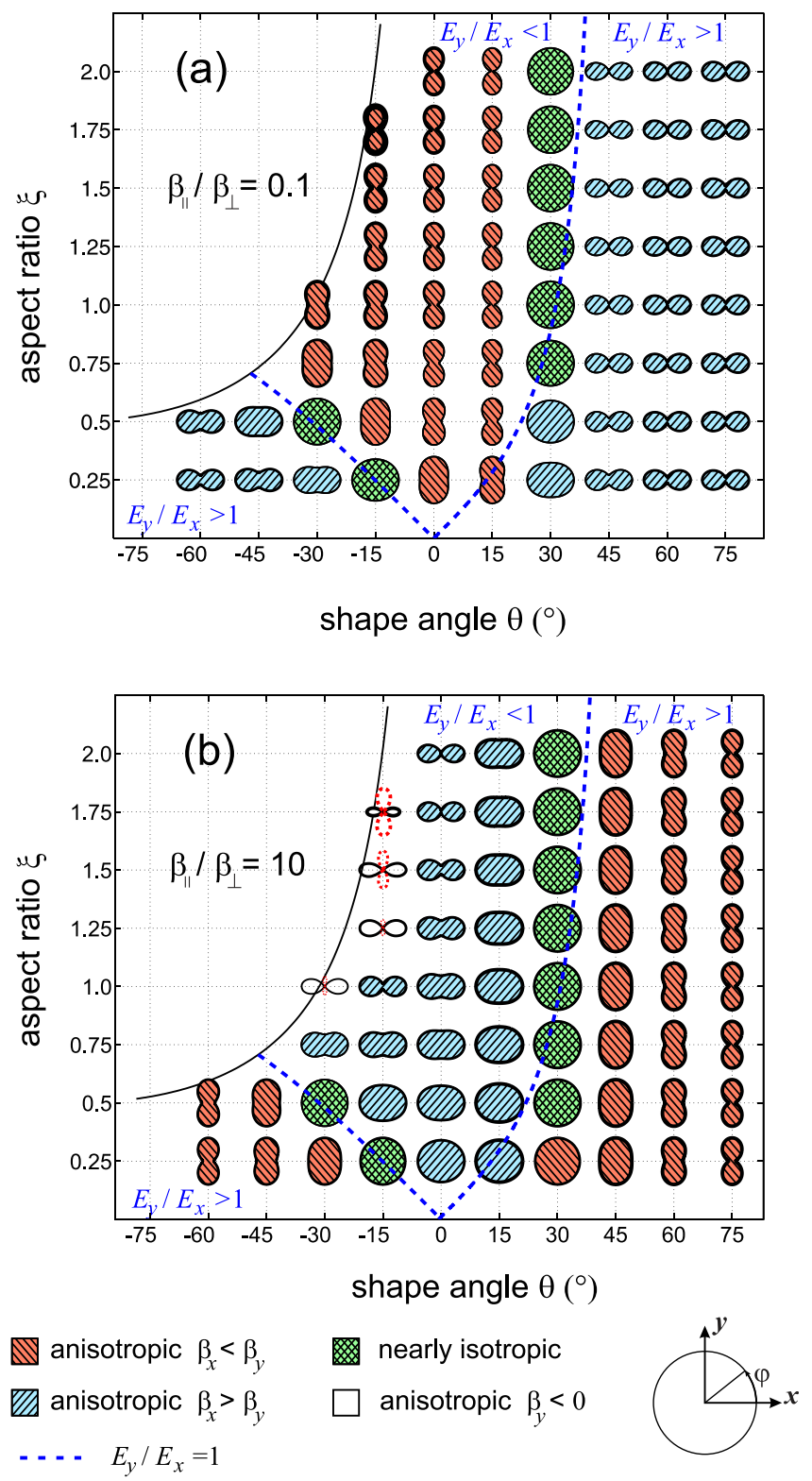

FIG. 2. Morphological phase diagram of the swelling anisotropy of honeycombs, presented in polar plots, where the control parameters are the shape angle $\theta$ and the aspect ratio $\xi$. (a) $\beta_{\|} / \beta_{\perp}=0.1$ and (b) $\beta_{\|} / \beta_{\perp}=10$. The line thickness represents the relative magnitude of swelling coefficients. The negative values are shown with dotted red lines. 
behavior of $\beta_{\varphi}$ can be categorized into four groups as shown in these figures: (1) the swelling is preferential in $x$ direction (right-hatch or blue), (2) the swelling is preferential in $y$ direction (left-hatch or red), (3) the swelling behavior is nearly isotropic (cross-hatch or green), and (4) configurations with negative swelling coefficient in one direction (white). In this case, the negative values are shown with dotted red lines. In these figures, the thickness of border lines is illustrated proportional to the magnitude of $\beta_{\varphi}$.

These two maps show how the geometrical parameters of cellular structures and the anisotropy in the swelling properties of the cell wall have a significant impact on the overall anisotropic swelling behavior of cellular materials. In order to explain the trends observed in this figure, we first look at the anisotropy in the elastic properties. The ratio of elastic moduli in $x$ and $y$ directions can be calculated following the simple relation derived from Gibson and Ashby model: ${ }^{2}$

$$
\frac{E_{y}}{E_{x}}=\frac{(1 / \xi+\sin \theta)^{2} \sin ^{2} \theta}{\cos ^{4} \theta} .
$$

This ratio switches from larger to smaller than 1 at a specific shape angle $\theta$ for each aspect ratio $\xi$. For example, when $\xi=1$, for $\theta>30^{\circ}$ the ratio is $E_{y} / E_{x}>1$, and for $\theta<$ $30^{\circ}$ the ratio is $E_{y} / E_{x}<1$. The loci of $\xi$ in which $E_{y} / E_{x}=1$ are derived from Eq. (3) and are plotted as a function of $\theta$ in Fig. 2 (blue dashed lines). These two curves are defined by $\xi=-\sin \theta$ for $-45^{\circ}<\theta<0^{\circ}$ and $\xi=\sin \theta \sec 2 \theta$ when $0^{\circ}<\theta<45^{\circ}$. As can be seen in this figure, the swelling behavior close to these curves is almost isotropic. One can assert that swelling acts as a set of internal forces that push the material qualitatively similarly to external forces. Based on this interpretation, the ratio $\beta_{\|} / \beta_{\perp}$ determines the contribution of the forces transmitted in $x$ and $y$ directions. So when the anisotropy switches from left-hatch (red) to righthatch (blue) pattern in Fig. 2, this is in some measure evidence of this assertion. A unit area of material may expand anisotropically because of two reasons: it may be acted on by forces whose distribution is anisotropic or its ability to resist force may be anisotropic. ${ }^{19}$ Under a uniform increase of the moisture content, if the cell walls of the honeycombs swell isotropically, the macroscopic swelling response is also isotropic, and there would be no internal forces. On the other hand, if the swelling properties of the cell wall are anisotropic, the mismatch between the material orientations at the cell corners which acts on the rigid cell connections provide the structure with a capacity to bear internal loads in an anisotropic way even when there is no constraint which results in macroscopic anisotropic swelling deformation. The increase of the swelling anisotropy can be tracked when the swelling map changes from circular to dumbbell shape. The anisotropy increases by increasing the aspect ratio $\xi$ for $\beta_{\|} / \beta_{\perp}=0.1$ in the left-hatch (red) regions and also for $\beta_{\|} / \beta_{\perp}=10$ in the right-hatch (blue) regions. Furthermore, the swelling phase diagrams obtained for $\beta_{\|} / \beta_{\perp}=0.1$ and $\beta_{\|} / \beta_{\perp}=10$ exhibit almost opposite trends.

The above study can be employed to explain the mechanism of swelling anisotropy in biological materials with honeycomb microstructure. In the cellular tissues of the biological materials, the swelling of the cell wall in normal direction is usually larger than in parallel direction, i.e., $\beta_{\|}<$ $\beta_{\perp}$ (Fig. 2(a)). Here we compare the trends predicted in Fig. 2(a) with two natural materials with cellular microstructure: earlywood cells in softwood and keel cells in ice plant. Softwoods are made up mainly of tracheids, which make up the bulk of the cells and have a roughly honeycomb-like structure. ${ }^{20}$ In Norway Spruce softwood, the shape angle of earlywood cells is in the range $\theta \simeq 0^{\circ}-15^{\circ}$, and the aspect ratio of cell wall lengths is about $\xi \simeq 0.5-2.0$. Wood cells swell in radial and tangential directions with respect to wood anatomical directions which are, respectively, equivalent to $x$ and $y$ directions in Fig. 1. Measurements by high-resolution synchroton X-ray tomographic microscopy reveal that earlywood radial strains are less than a third of its tangential strains. ${ }^{5}$ We observe in Fig. 2(a) that, for this range of $\theta$ and $\xi$, the swelling coefficient $\beta_{\varphi}$ in $y$ direction is larger than $x$ direction which is in line with experimental results. Another example is the origami-like hydration-dependent unfolding of ice plant seed capsules which stems from the swelling of the honeycomb-like keel cells. ${ }^{8}$ The shape angle of the keel cells is generally $\theta \geq 60^{\circ}$, and the aspect ratio is about $\xi \simeq 0.5$. From the swelling map presented in Fig. 2(a), we expect that, in contrary to wood cells, swelling to be more significant in $x$ direction. Experimental measurements show that, during the swelling, the cells deform anisotropically in the transverse plane. This anisotropic deformation at the cell level upon swelling results in the flexing movement of the keel, which requires a large deformation in the $x$ direction. $^{8}$ Transverse cell shape change in $x$ direction, from dry to wet state, is fourfold larger than in $y$ direction which confirms the trend observed in our simulation results. These two examples clearly show how the microscopic geometrical parameters of biological tissues in combination with anisotropy in swelling behavior of the cell walls determine the overall response of the medium. In honeycombs, for regular hexagons $\left(\theta=30^{\circ}, \xi=1.0\right)$, the deformation of the cell walls is mainly axial while irregular honeycombs may undergo a combination of axial and flexural deformation depending on the ratio of $\beta_{\|} / \beta_{\perp}$ and the geometry of honeycombs. However, when $\beta_{\|} \ll \beta_{\perp}$, the flexural deformation becomes more significant specifically at cell corners. Considering the

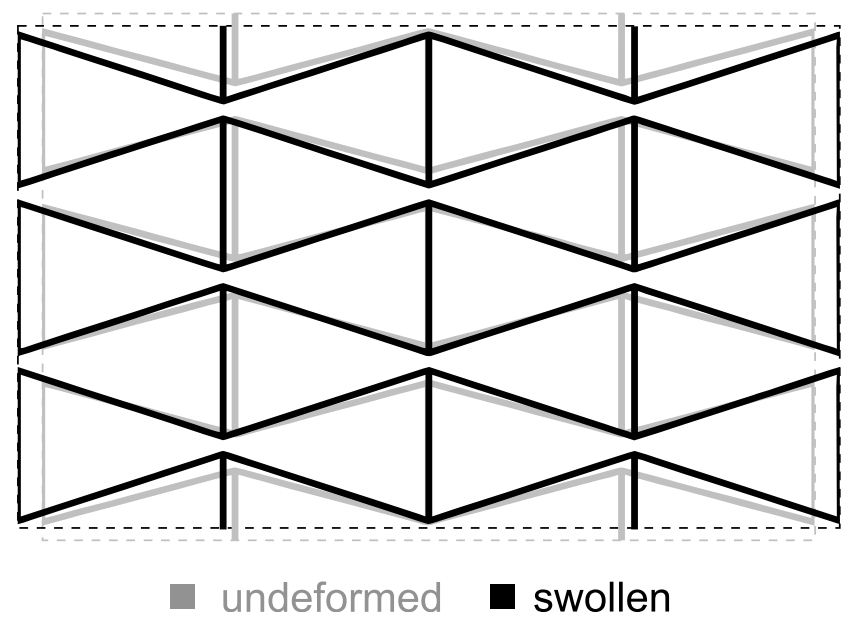

FIG. 3. Undeformed and swollen states of a re-entrant honeycomb cellular structure with $\theta=-15^{\circ}, \xi=1.5$, and $\beta_{\|} / \beta_{\perp}=10$. The sample swells in $x$ direction and shrinks in $y$ direction. 

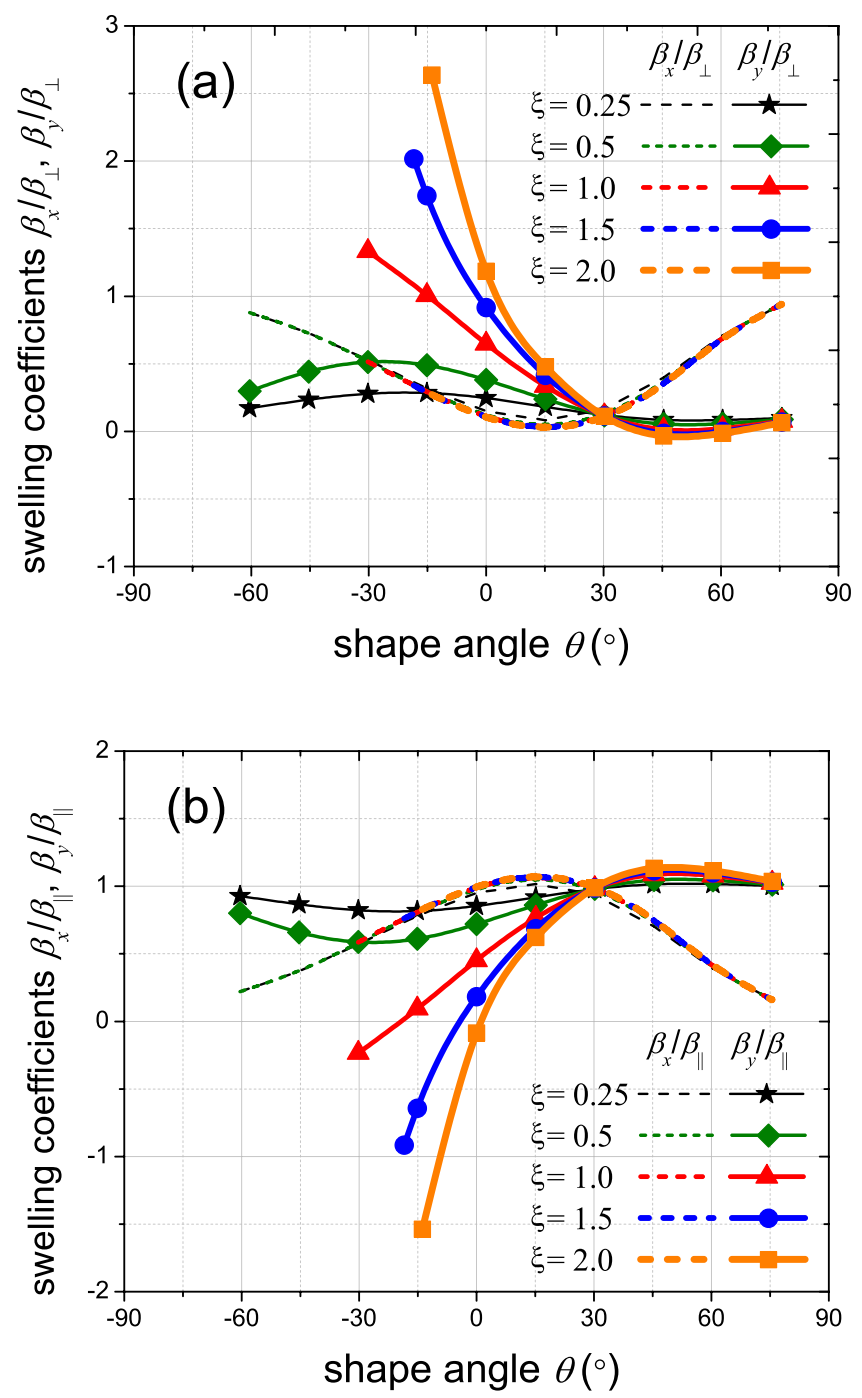

FIG. 4. Swelling coefficients $\beta_{x}$ and $\beta_{y}$ of honeycombs as a function of shape angle $\theta$ for different aspect ratios $\xi$ and $t / h=0.05$ when (a) $\beta_{\|} / \beta_{\perp}=$ 0.1 and (b) $\beta_{\|} / \beta_{\perp}=10$.

above two examples, we observe that the variation of shape angle from $\theta<30^{\circ}$ to $\theta>30^{\circ}$ results in a total different deformation pattern in earlywood and keel cells. Here, it should be mentioned that the macroscopic anisotropic swelling behavior of cellular materials originates from the swelling anisotropy of the cell wall material; otherwise, the influence of geometry is totally canceled out and the overall behavior becomes isotropic.

According to the swelling map presented in Fig. 2(b), in honeycombs with auxetic behavior $\left(\theta<0^{\circ}\right)$, for large $\xi$, the moisture induced tensile deformation in the cell walls activates the re-entrant mechanism which results in shrinkage of the structure in $y$ direction. Fig. 3 shows the swelling deformation of a honeycomb structure where the geometrical parameters are $\theta=-15^{\circ}, \xi=1.5$, and the swelling coefficient of the cell wall is larger in parallel direction, i.e., $\beta_{\|} / \beta_{\perp}=10$. It can be seen that this structure simultaneously swells in $x$ direction and shrinks in $y$ direction.

The swelling coefficients of cellular structures ranging from conventional to re-entrant honeycombs are computed as a function of shape angle $\theta$ for different aspect ratios $\xi$.
The results for $\beta_{\|} / \beta_{\perp}=0.1$ and $\beta_{\|} / \beta_{\perp}=10$ are, respectively, shown in Figs. 4(a) and 4(b). These figures show that $\beta_{x}$ is not sensitive to aspect ratio $\xi$ and follows almost a single curve. This trend is observed whether the swelling behavior of the cell walls is larger in normal or parallel directions. On the other hand, $\beta_{y}$ is strongly dependent on $\xi$ specifically for $\theta<30^{\circ}$ which provides the material with tunable swelling behavior in $y$ direction. For cell walls with larger normal swelling behavior, $\beta_{y}$ goes to very high positive values while for those with larger parallel swelling, it goes to high negative values. Therefore, it is possible to design cellular structures which can swell much more than their constituents can do. In addition, we can propose cellular microstructures which might shrink when they absorb moisture. For a regular hexagon, the swelling behavior is isotropic irrespective of the anisotropy of the swelling of the cell wall material, but the swelling coefficient is larger when $\beta_{\|}>\beta_{\perp}$.

In conclusion, we showed that the macroscopic swelling behavior of cellular materials can be controlled by adjusting the geometrical parameters of the structure and the swelling anisotropy of the cell wall material. We presented the anisotropy phase diagram for the swelling behavior of periodic cellular materials for honeycombs. It is found that the swelling anisotropy depends strongly on the anisotropy of the macroscopic elastic properties of the cellular materials. The results of this research can be used for studying the hygromechanical behavior of biological materials. The knowledge gained from these fundamental studies can be reflected to the development and design of new bio-inspired resilient and durable structural materials.

The authors are grateful for the financial support of the Swiss National Science Foundation (SNF) under Grant No. 125184.

${ }^{1}$ R. S. Lakes, Nature 361, 511 (1993).

${ }^{2}$ L. J. Gibson and M. F. Ashby, Cellular Solids: Structure and Properties, 2nd ed. (Cambridge University Press, 1997).

${ }^{3}$ L. Salmen, C. R. Biol. 327, 873 (2004).

${ }^{4}$ L. Salmen and I. Burgert, Holzforschung 63, 121 (2008).

${ }^{5}$ D. Derome, M. Griffa, M. Koebel, and J. Carmeliet, J. Struct. Biol. 173, 180 (2011).

${ }^{6}$ R. J. Redgwell, E. MacRae, I. Hallett, M. Fischer, J. Perry, and R. Harker, Planta 203, 162 (1997).

${ }^{7}$ R. Elbaum, S. Gorb, and P. Fratzl, J. Struct. Biol. 164, 101 (2008).

${ }^{8}$ M. J. Harrington, K. Razghandi, F. Ditsch, L. Guiducci, M. Rueggeberg, J. W. Dunlop, P. Fratzl, C. Neinhuis, and I. Burgert, Nat. Commun. 2, 337 (2011).

${ }^{9}$ P. Gillis, Wood Sci. Technol. 6, 138 (1972).

${ }^{10}$ L. J. Gibson, M. F. Ashby, G. S. Schajer, and C. I. Robertson, Proc. R. Soc. Lond. A 382(1782), 25-42 (1982).

${ }^{11}$ A. G. Kolpakov, J. Appl. Math. Mech. 49, 739 (1985).

${ }^{12}$ K. E. Evans and A. Alderson, Adv. Mater. 12, 617 (2000).

${ }^{13}$ R. S. Lakes, J. Mater. Sci. Lett. 15, 475 (1996), available at http://link. springer.com/article/10.1007\%2FBF00275406?LI=true\#.

${ }^{14}$ R. S. Lakes, Appl. Phys. Lett. 90, 221905 (2007).

${ }^{15}$ A. Rafsanjani, D. Derome, and J. Carmeliet, Compos. Struct. 95, 607 (2013).

${ }^{16}$ R. Hill, J. Mech. Phys. Solids 11, 357 (1963).

${ }^{17}$ Y. Ishimaru, K. Arai, M. Mizutani, K. Oshima, and I. Iida, J. Wood Sci. 47, 185 (2001).

${ }^{18}$ A. Rafsanjani, D. Derome, and J. Carmeliet, Mech. Mater. 55, 49 (2012).

${ }^{19}$ T. I. Baskin, Annu. Rev. Cell Dev. Biol. 21, 203 (2005).

${ }^{20}$ L. J. Gibson, J. R. Soc., Interface 9(76), 2749 (2012). 